\title{
Surgical treatment and outcome of cerebral cavernomas - a 10 years' experience
}

\author{
A. Giovani, Aurelia Sandu, Angela Neacsu, R.M. Gorgan
}

\begin{abstract}
Cavernous malformations (cavernomas) are congenital low flow angiographic occult vascular lesions with a high tencency to bleeding. The prevalence of cerebral cavernous vascular malformations is estimated to be $0.4 \%$ to $0.9 \% .2,7 \mathrm{CMs}$ in deep locations, including the brainstem, thalamus, and basal ganglia, account for $9 \%$ to $35 \%$ of all malformations in the brain. We performed a retrospective 10 years study on 130 operated cerebral cavernomas and discussed the clinical status at presentation, the choice and timing of the surgical approach and the short and longterm follow up. The 130 operated cases were divided into a supratentorial 102 (78.46\%) group and an infratantorial group $28(21.53 \%)$. The average age at presentation was 43,62 years old $(17-76)$ and there was no sex predominance, male/female $=1,44$ (77/53). Only in 14 cases (10.76\%) we could find multiple cavernomas which had relatives with multiple cavrnomas, but the familial inheritance was not studied, and only the symptomatic lesion was resected. We divided the outcome results reporting for agroup with superficial respectively profound lesions. The long term follow up for the patients in the profound lesions group showed that $31 / 37(83,78 \%)$ of patients had a mRS between 0 and 2 , and the rest had a poor long term outcome. After surgery there was no clinical deterioration in the superficial lesions group and 22 patients from those who presented in mRS 2 showed neurological improvement on long term follow up, meaning that $82,79 \%$ of patients had a 0 or $1 \mathrm{mRS}$. Microsurgery is the treatment of choice in symptomatic brain cavernomas, total resection being the only curative treatment, capable to prevent further bleeding and to offer an efficient control of seizures.
\end{abstract}

Key words: cavernous malformation, microneurosurgery, seizures.

\section{Introduction}

Cavernous malformations (cavernomas) are congenital low flow vascular lesions, which can be associated with other lesions from the same spectrum like capillary telangiectasias and developmental venous anomalies, they account for $1 \%$ of intracranial vascular lesions an $\mathrm{d} 15 \%$ of vascular malformations. $(1,6)$ The prevalence of cerebral cavernous vascular malformations is estimated to be $0.4 \%$ to $0.9 \%$ $(2,7)$. Malformations in deep locations 
including the brainstem, thalamus, and basal ganglia are of particular interest because their critical location renders them very challenging for surgical resection $(3,7,10)$. Cavernomas are well circumscribed, benign, low-flow vascular malformations, composed of irregular sinusoidal vascular channels, lacking smooth muscle and elastic fibers.

They lack feeding arteries or draining veins and contain no neural tissue. By comparison to other vascular malformations included in McCormick classification (1966), angiography is not a good diagnostic tool as there is no blood flow inside these lesions, yet their characteristic mulberry shape on MRI directs the diagnostic. $(8,9,10)$

Though the mechanism of growth of these lesions was not completely elucidated most studies sugest that repeated subclinical bleeding episodes inside the lesion and the subsequent thrombus formation and trombolisis create the "caverne" that give the name and the typical mulberry appearance on T2 MRI. (5)

After Rigamonti's first description of this aspect in 1986, Zabramsky developed a 4 grade classification of cavernous malformations based on MRI, in which grade lare hyperintense grade II are both hyper and hypointense, grade III are hypointense and grade IV are small "black spot lesions" visible only on gradient echo these were considered capillary telangiectasies. $(21,25)$

CMin deep locations is not completely understood, but most reports suggest that they have a higher rate of hemorrhage than superficial CMs as well as high rebleed rates. (2)

Treatment options include observation, and surgery, and a few authors in the past reported cases treated with radiosurgery but now it gained acceptance that radiosurgery has no positive long term impact in the treatment of cavernomas. Especially superficial supratentorial lesions with a low risk of bleeding and producing neurologic deficit can be followed by observation alone but this approach is not safe for patients harboring deep seated lesions prone to produce new neurological deficits in case of rerupture. Most of the studies report good outcomes with almost $100 \%$ complete removal, though this is not the case in the posterior fossa where the postoperative morbidity can be as high as $30 \%$. (4)

\section{Materials and methods}

The objectives of the study were to analyze the the factors that influence the short and long term outcome, to establish the best indications for surgery and to define the safe corridors to approach deep seated lesions. We retrospectively reviewed the clinical records the surgical details and the acute and delayed surgical complications for 130 consecutive patients operated between January 2000 and December 2009 in our department. The average follow up was around 5 years (between 6 months and 10 years). All the patients were investigated with $\mathrm{CT}$ scan and MRI as the cavernomas are angiographically occult lesions. The outcome was evaluated comparing preoperative with postoperative modified Rankin Scale (mRS).

The senior surgeon (R.M.G) did not feel the need to use neuronavigation or of Stereotaxy for locating the lesions nor for those located immediately subcortical or for 
DOI: 10.2478/romneu-2014-0055

those in deep locations. We did not use motor, sensitive or cranial nerves evoked potentials. Based on the information from the MRI the surgical approach was directed on the shortest way to the lesion. The indication for surgery depends on the clinical state of the patient, for the supratentorial location either repeated seizures, motor or sensory deficit if the internal capsule or the thalamus are injured or visual disturbance if the optic radiations are injured. $(20,22)$ As the natural course of the brainstem cavernomas is more aggressive than of those located supratentorial and the risk of clinical significant hemorrhage is higher surgical resection is indicated in all symptomatic and accessible lesions. Even if some studies report a $30 \%$ risk of new neurological deficit after surgery these make often a significant clinical recovery and there is no risk of rebleeding. $(6,8,14)$

In almost all subcortical and deep seated supratentorial lesions the approach was transcortical with care not to injure eloquent areas or important veins, but respecting the rule of the shortest way to the lesion. The approaches used for the infratentorial lesions including those of the brainstem were retrosigmoid, supracerebelar infratentorial, suboccipital and suboccipital telovelar. All the lesions were approached from the point where they came closest to the surface (the 2 point method). $(26,27)$

The microsurgical technique included sharp dissection and piecemeal resection or one piece resection where possible in more superficial lesions. Except for the deep seated lesions the surrounding hemosiderin rich gliotic ring was also removed, but the associated venous anomalies where it was the case were all left untouched.

In cases with multiple cavernomas surgery is indicated for the lesions responsible for neurological deficit, the other lesions are just imagistically followed. (7)

\section{Results}

Between 2000 and 2009130 consecutive patients with cavernomas were operated in our department. The mean age at presentation was 43,64 years range between 17 and 76 .

The 130 operated cases were divided into a supratentorial $102(78.46 \%)$ group and an infratantorial group $28(21.53 \%)$. The average age at presentation was 43,62 years old (17-76) and there was no sex predominance, male/female $=1,44(77 / 53)$. Only in 14 cases (10.76\%) we could find multiple cavernomas which had relatives with multiple cavrnomas, but the familial inheritance was not studied, but only the symptomatic lesion was resected.

The supratentorial cavernomas were located in frontal lobes in 52 patients (40\%), in temporal lobes in 27 patients $(20.76 \%)$ in parietal lobes in 25 patients $(19,23 \%)$ in occipital lobes in 2 patients $(1,53 \%)$ and deep in $24(18,46 \%)$

The infratentorial cavernomas were distributed in cerebellum 15 patients (53.57\%), and in brainstem and cerebellar peduncles in 13 patients $(46,42 \%)$. Out of these 13 patients, 3 were in medulla, 2 in the pontomedullary junction 4 in the pons and 4 in the mesencephalus.

The clinical presentation was differentin the two groups: in the supratentorial one, seizures and headache while the dominant 
symptoms but in the infratentorial group where the cranial nerve deficits, hemiparesis, numbness and cerebellar syndrome were dominant (Table 1).

Hemorrhage was present on MRI in 40 patients (30.76\%) in 38 (95\%) as intraparenchymal hematoma, in 1 patient as intraventricular bleeding and in 1 as subarachnoid hemorrhage. 31 of these patients $(77.5 \%)$ were in a poor preoperative mRS, having an altered mental state.

The patients with seizures $66 \mathrm{p}$ (50.76\%) were stratified according to type and frequency (Table 2).

TABLE I

\begin{tabular}{|l|l|l|l|l|l|l|}
\hline Supratentorial cavernomas \\
\hline Hemorrhage & Seizures & Headache & Hemiparesis & Numness & Aphasia & Visual deficits \\
\hline $29 / 102(28,43 \%)$ & $\begin{array}{l}66 / 102 \\
(64.70 \%)\end{array}$ & $\begin{array}{l}58 / 102 \\
(56,86 \%)\end{array}$ & $\begin{array}{l}19 / 102 \\
(18,62 \%)\end{array}$ & $\begin{array}{l}19 / 102 \\
(18,62 \%)\end{array}$ & $\begin{array}{l}13 / 102 \\
(12.74 \%)\end{array}$ & $3 / 102(2.94 \%)$ \\
\hline
\end{tabular}

\section{TABLE II}

\begin{tabular}{|cl|}
\hline Type & \\
\hline$\bullet$ & Generalized tonic-clonic seizures $-32 \mathrm{p}(48.48 \%)$ \\
\hline$\bullet$ & Partial complexe seizures $-24 \mathrm{p}(36,36 \%)$ \\
\hline$\bullet$ & Partial simple seizures $-15 \mathrm{p}(22.72 \%)$ \\
\hline$\bullet$ & Partial seizure, secondary generalized $-9 \mathrm{p}(13.63 \%)$ \\
\hline Frequency & ^ some patients presented more than one type of seizures \\
\hline$\bullet$ & $1-2$ seizures before surgery $-30 \mathrm{p}(45,45 \%)$ \\
\hline$\bullet$ & $1-2$ seizures/year $-17(25,75 \%)$ \\
\hline$\bullet$ & 1 seizure/week - $9(13.63 \%)$ \\
\hline$\bullet$ & daily seizures - $11(16.66 \%)$ \\
\hline & \\
\hline $11 \mathrm{p}(16.66 \%)$ presented refractory seizures \\
\hline Mean time between seizure onset and surgery $=4$ mo $(0.4 \rightarrow 40 \mathrm{mo})$ \\
\hline
\end{tabular}

Based on this data we identified a few risk factors for seizure reccurence. There were no seizures in deep locations. 
TABLE III

\begin{tabular}{|cl|}
\hline \multicolumn{2}{|l|}{ Risk factors for seizures occurrence: } \\
\hline$\bullet$ & Cortical location \\
\hline$\bullet$ & T location, especially temporomesial location \\
\hline$\bullet$ & Large lesions, including hemosiderinic ring \\
\hline$\bullet$ & Focal neurological deficits \\
\hline$\bullet$ & Left side cavernomas \\
\hline$\bullet$ & Acute hemorrhage \\
\hline$\bullet$ & Subacute hemorrhage \\
\hline were not confirmed as risk factors for seizures occurrence \\
\hline$\bullet$ & Sex, \\
\hline$\bullet$ & age, \\
\hline$\bullet$ & diameter of hemosiderinic ring, \\
\hline$\bullet$ & diameter of surrounding edema, \\
\hline$\bullet$ & multiple lesions \\
\hline
\end{tabular}

\section{Outcome}

When we analyzed the outcome we studied the group of corticalised or superficial subcortical cavernomas and that of deep seated lesions, in brainstem, thalamus and basal ganglia.

Total resection was performed in all cases, but the surrounding hemosiderin ring was not removed in deep seated lesions where such a maneuver could harm grey matter nuclei and no mortality was registered on short or long term follow up in this series of 130 patients.

Both Karnovsky and mRS improved after surgery. In patients with deep seated lesions (37 patients), $12(32,43 \%)$ presented in a poor preoperative state $\mathrm{mRS}>2.10$ patients from this group had worsened deficits immediately after surgery, 4 of these in brainstem cavernomas but all of them showed a good recovery with follow up 5 of them remained in mRS 2 and 3 with $m R S>2$. The complications included worsened hemiparesis, worsened ataxia, $7^{\text {th }}$ nerve paresis and VI or III ${ }^{\text {rd }}$ nerve paresis.

The immediate postoperative status for this group was excellent in $10 / 37(27,02 \%)$ of patients, good in $13 / 37(35,13 \%)$ patients and poor in $14(37,83 \%)$ patients. The long term follow up for these patients showed that $31 / 37$ $(83,78 \%)$ of patients had a mRS between 0 and 2 , and the rest had a poor long term outcome.

The situation was different in the group of superficial cavernomas where 55/93 (59.13\%) of patients presented with a mRS of 0 or 1 and $36 / 93(38,7 \%)$ presented with $\mathrm{mRS}$ of 2 and 2 patients with an $\mathrm{mRS}>2$.

After surgery there was no clinical deterioration in this group and 22 patients from those who presented in mRS 2 showed neurological improvement on long term follow up, meaning that $82,79 \%$ of patients had a 0 or $1 \mathrm{mRS}$. 


\section{Case presentations}

Case 1: A 70 years old male was admitted for persistent headache and left homonymous hemianopsia that worsened in the week previous presentation.

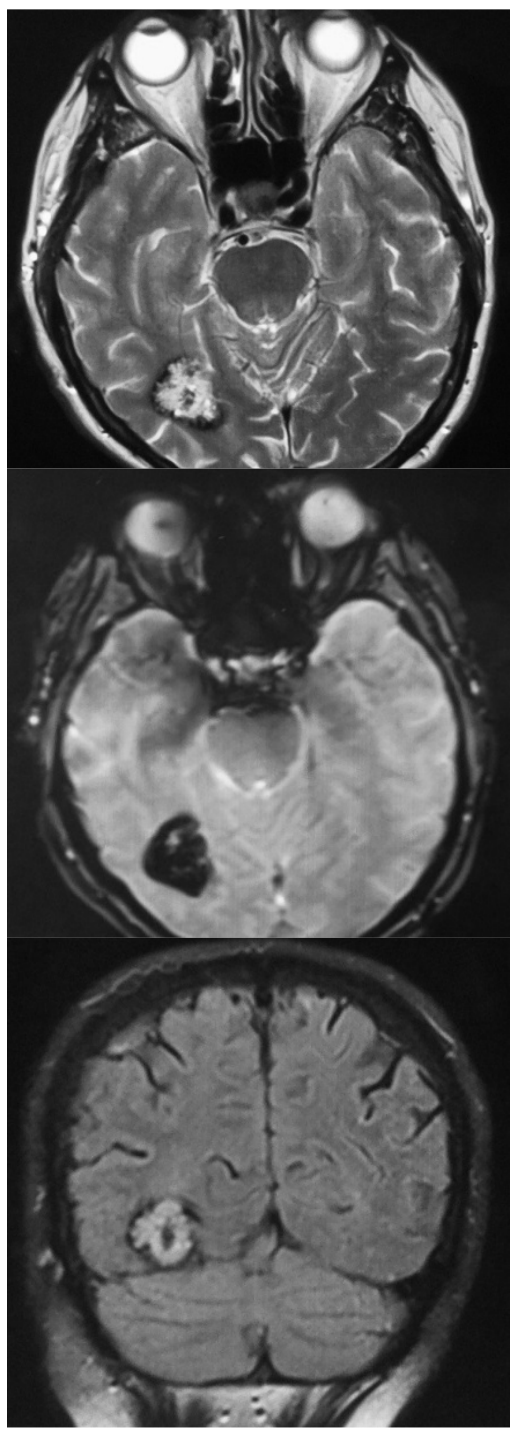

Figure 1 - MRI - right occipital heterogenous lesion, with salt and pepper appearance suggestive for cavernoma
The tumor was completely removed with the surrounding hemosiderin ring using a right suboccipital occipital-supratentorial approach. Using the gravity with no need for mechanical retraction, the lesion was exposed corticalised on the tentorial surface of the right occipital lobe. The tumor was piecemeal removed and then the hemosiderin ring was resected. Hemostasis was achieved with bipolar and intensive lavage with serum. After the dural closure the scalp is closed on epidural drain. The postoperative CT scan confirmed complete removal.

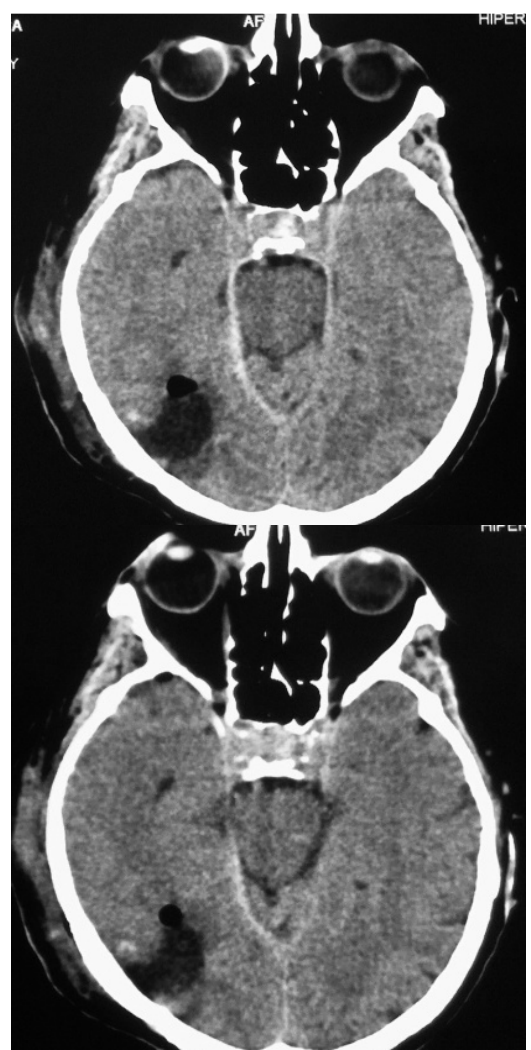

Figure 2 - Same patient with postop CT images of the occipital cavernoma - total removal 
Case 2: A 45 years old female presented with altered mental state (14 p) and a history of headache, vomiting, swallowing disorders (liquid and solid food), gait disturbances (astasia-abasia), mild right faciobrachial paresis with onset 7 days before admission. The Neurologic exam showed mild right faciobrachial paresis and IX, X, XI and XII cranial nerves paresis.

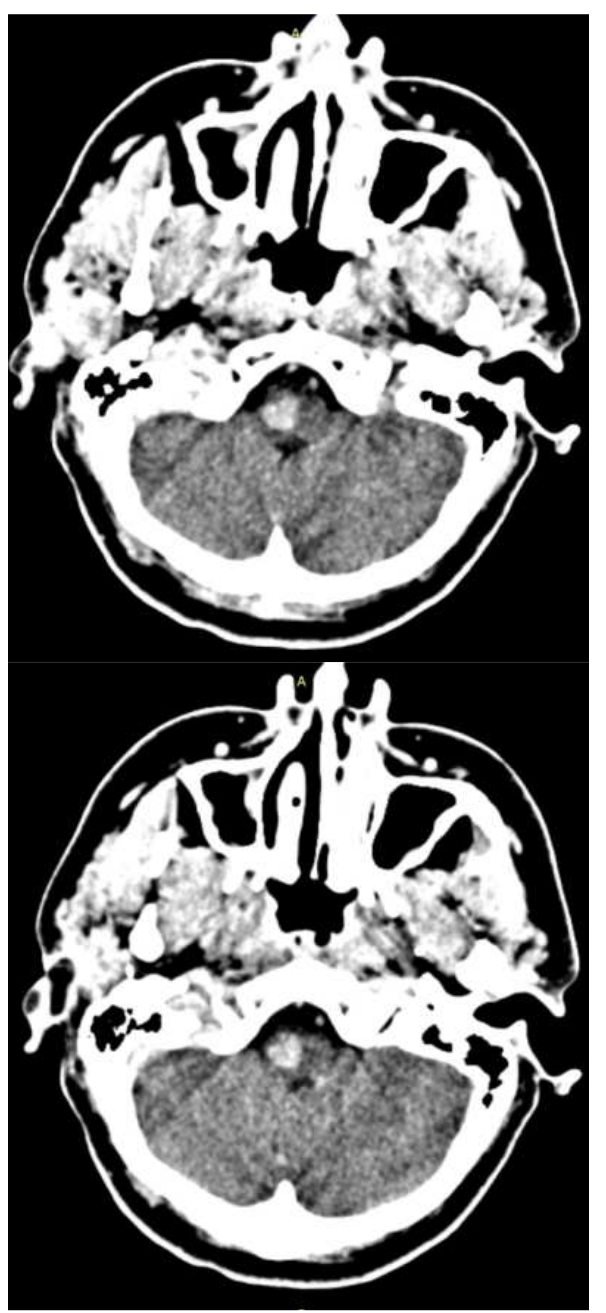

Figure 3 - CT scan - spontaneous hyperdense lesion within the right medulla, $\varnothing=13 \mathrm{~mm}$
DOI: 10.2478/romneu-2014-0055

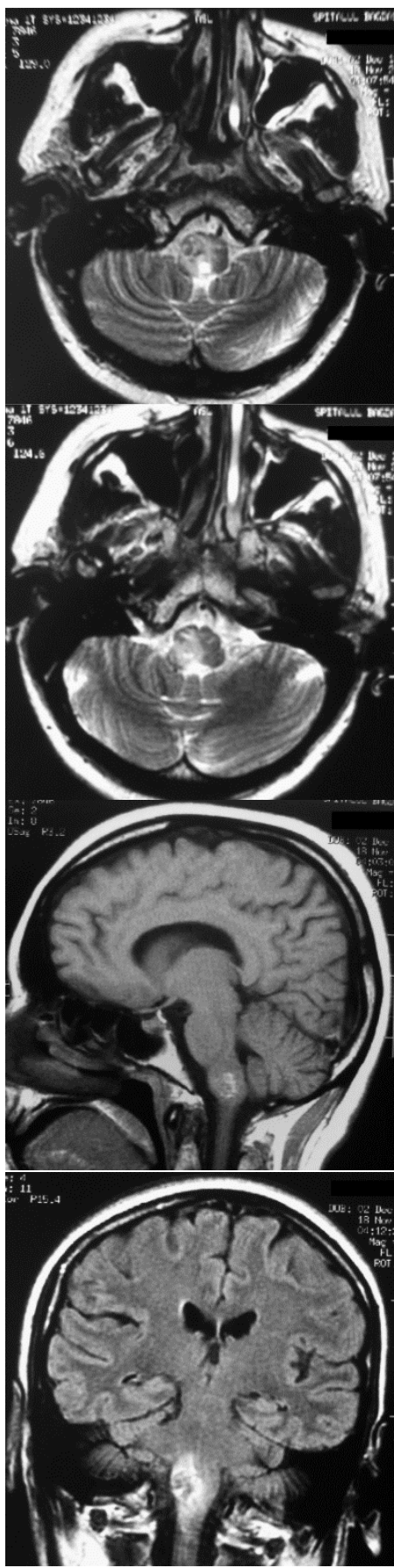

Figure 4 - MRI: right medulla lesion, $\mathrm{H}$-iso in T1 and h-HT2, $\varnothing=13 \mathrm{~mm}$, suggestive for cavernoma, with subacute bleeding 
The lesion was completely resected using a telovelar approach. A midline suboccipital craniectomy was performed, and after opening the cisterna magna a telovellar approach is used to access the medulla. As the lesion appeared subpial it was entered directly and then piecemeal resected completely. The surrounding yellow gliotic plane was left intact. Dura mater was reconstructed with Kolagen and then with the drain left in place the muscles plane and the skin were closed.

The outcome was favorable with partial remission of the cranial nerves deficits and improvement of swallowing disorders at 2 months follow up. (Figure 4)

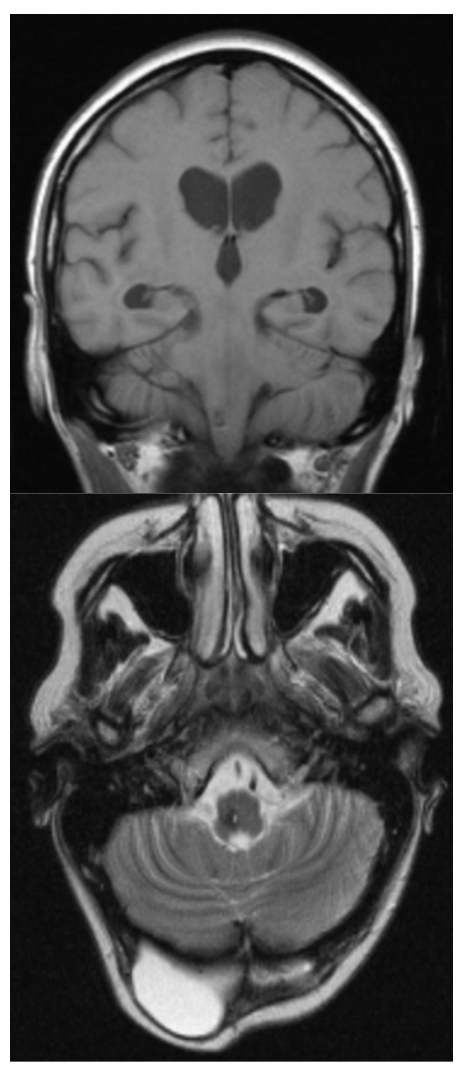

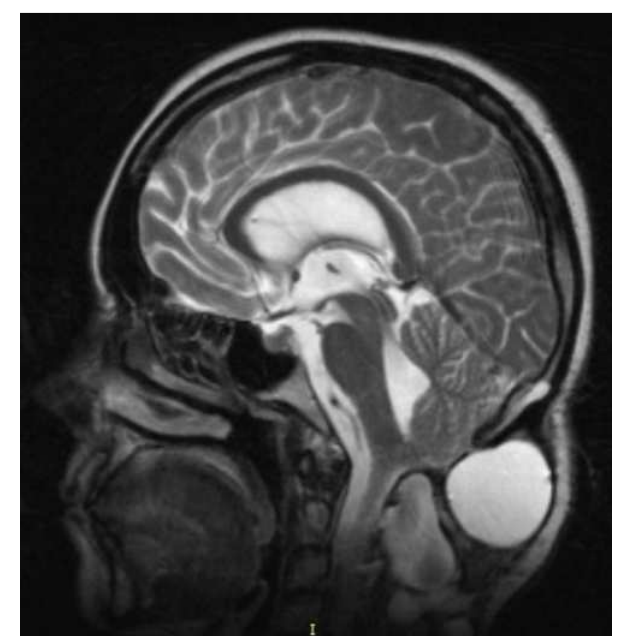

Figure 5 - Postop. MRI with complete resection of the cavernoma

\section{Discussions}

The frequency of asymptomatic cavernomas can be as high as $40 \%$ (Zabramsky), but the actual data is impossible to quantify.

The most common presenting symptoms for the supratentorial group were seizures, headache, hemiparesis and numbnes. The most common infratentorial presentation were cranial nerve deficits followed by hemiparesis and numbness and cerebellar symptoms.

Compared to the infratentorial group where the hemorrhagic presentation was almost the rule (93\%) only $39 \%$ of the supratentorial group patients had this presentation.

The division into supratentorial and infratentorial groups is not enough and further studies should focus on the differences of the groups of patients with cortical or subcortical cavernomas compared to those 
DOI: 10.2478/romneu-2014-0055

with cavernomas seated in brainstem, thalamus or basal ganglia. $(1,3)$

The best method for preoperative and postoperative investigating the patient is the MRI. It can show the borders of the lesion the intra or extralesional hemorrhage and the associated edema and also is invaluable in planning the aproach especially if neuronavigation or stereotaxy are not used for localization. Angiography can be useful in defining associated lesions because cavernomas are not visible on it but we did not make a standard from using it and this may be a reason why venous associated anomalies escaped our view.

As cavernomas in deep locations have an annual hemorrhage rate of $5,1 \%$ and a rebleed rate of $31,5 \%$ per patient per year, there was no time for wait and see in these cases. Observation was reserved only to those cases we considered inoperable. Complete removal was achieved in all cases but with the cost of new neurological deficits in 4 patients (3\%), all of these presenting deep seated lesions. The postoperative mRS was good or excellent in $97 \%$ of patients compared to $64 \%$ preoperative and was even more evident in patients with deep seated lesions. The control of the seizures was achieved in all patients who presented with seizures, and almost $60 \%$ of them were free of medication after surgery.

The dimensions of the lesions were not statistically different in the two groups but in the infratentorial group they correlated with the hemorrhage and neurologic deficit. Some studies report larger sizes of cavernomas in children but our study included only adults. We could find an association with venous anomalies only in 3 patients which is in contrast to the reports of other studies.

As prospective studies are lacking the hemorrhage rates which are the key to the surgical indication are calculated based on retrospective analysis considering cavernomas as congenital lesions, yet more and more studies document the appearance of de novo cavernomas in sporadic or familial cases. Even the results of different studies differ it became clear that once symptomatic the cavernomas have a high rate of rebleeding causing new neurological deficits. $(14,18,19)$

Because of their tendency to bleed cavernomas are always in the surgical focus, yet surgery should not be proposed for all lesions, most of the supratentorial lesions can be treated conservatively if asymptomatic. Most of the time the hemorrhage is inside the cavrnoma causing it's enlargement in time, but when seated in deep nuclei even this enlargement can give neurological deficit, acting as a mass effect. When located in eloquent areas even small hemorrhage outside the lesion can be echoed in severe deficits. (11, 12)

Our study aimed at identifying those lesions with a higher tendency to produce deficits for which surgery is the best and the safest treatment but as is the case with many studies on cavernomas it is limited by its retrospective nature.

As The most frequent manifestations of supratentorial lesions are repeated seizures, which disturb the patient's life balance we identified a few risk factors for seizures like the cortical and more frequently the temporomesial location, the large cavernomas, 
the location in the left hemisphere, the acute or subacute hemorrhage and edema. In all supratentorial lesions we removed the hemosiderin ring as well and we believe this is one of the main reasons for the complete disappearance of seizures in most of the cases. $(23,24)$

We identified a few positive prognostic factors including a high preoperative Karnofsky score coincident with a mRS of 0 or 1 , the small size and the superficial location of the lesion.

We did not consider radiosurgery an alternative to deep seated lesions because we considered the high rates of rebleeding after radiosurgery inacceptable and many studies showed that cavernomas can occur after radiation therapy. (12)

\section{Conclusions}

Symptomatic deep cavernous malformations in the brainstem, basal ganglia, and thalamus have a high bleed and rebleed rate and an aggressive natural history. Early surgery provides excellent clinical results and protects against future hemorrhages.

Microsurgery is the treatment of choice in symptomatic brain cavernomas, total resection being the only curative treatment, capable to prevent further bleeding and to offer an efficient control of seizures.

Complete cavernoma resection and resection of surrounding hemosiderin is recommended except for cavernomas located in the brainstem or in eloquent areas

ACKNOWLEDGEMENT: This paper was co-financed from the European Social Fund, through the Sectorial Operational Programme Human Resources Development 2007-2013, project number POSDRU/159/1.5/S/138907 "Excellence in scientific interdisciplinary research, doctoral and postdoctoral, in the economic, social and medical fields EXCELIS", coordinator The Bucharest University of Economic Studies.

\section{Correspondence \\ Giovani Andrei \\ giovani.andrei@gmail.com}

\section{References}

1.Abdulrauf SI, Kaynar MY, Awad IA. A comparison of the clinical profile of cavernous malformations with and without associated venous malformations. Neurosurgery. 1999;44(1):41-46.

2.Abla AA, Lekovic GP, Garrett M, et al. Cavernous malformations of the brainstem presenting in childhood: surgical experience in 40 patients. Neurosurgery. 2010;67 (6):1589-1598; discussion 1598-1599.

3.Abla AA, Lekovic GP, Turner J, deOliveira JG, Porter R, Spetzler RF. Advances in the treatment and outcome of brainstem cavernous malformation surgery: a singlecenter case series of 300 surgically treated patients. Neurosurgery. 2011;68(2):403-414.

4.Casazza M, Broggi G, Franzini A, Avanzini G, Spreafico R, Bracchi M, Valentini MC: Supratentorial cavernous angiomas and epileptic seizures: Preoperative course and postoperative outcome. Neurosurgery 39:26-34, 1996. 5.Clatterbuck RE, Elmaci I, Rigamonti D: The nature and fate of punctate (type IV) cavernous malformations. Neurosurgery 49:26-32, 2001.

6.Cohen DS, Zubay GP, Goodman RR: Seizure outcome after lesionectomy for cavernous malformations. J Neurosurg 83:237-242, 1995.

7.Dandy WE. Venous abnormalities and angiomas of the brain. Arch Surg. 1928;17 (5):715-793.

8.Del Curling O Jr, Kelly DL Jr, Elster AD, Craven TE: An analysis of the natural history of cavernous angiomas. J Neurosurg 75:702-708, 1991.

9.Engel JJ: Outcome with respect to epileptic seizures, in Engel JJ (ed): Surgical Treatment of the Epilepsies. New 
DOI: 10.2478/romneu-2014-0055

York, Raven Press, 1987, pp 553-571.

10.Ferroli P, Casazza M, Marras C, Mendola C, Franzini A, Broggi G: Cerebral cavernomas and seizures: A retrospective study on 163 patients who underwent pure lesionectomy. Neurol Sci 26:390-394, 2006.

11.Ferroli P, Sinisi M, Franzini A, Giombini S, Solero CL, Broggi G. Brainstem cavernomas: Long-term results of microsurgical resection in 52 patients. Neurosurgery. 2005;56(6):1203-1212.

12.Hasegawa T, McInerney J, Kondziolka D, Lee JY, Flickinger JC, Lunsford LD. Long-term results after stereotactic radiosurgery for patients with cavernous malformations. Neurosurgery. 2002;50(6):1190-1197.

13.Kondziolka D, Lunsford LD, Kestle JR. The natural history of cerebral cavernous malformations. J Neurosurg. 1995;83(5):820-824.

14.Kupersmith MJ, Kalish H, Epstein F, et al. Natural history of brainstem cavernous malformations. Neurosurgery. 2001;48(1):47-53.

15. Mathiesen T, Edner G, Kihlstrom L. Deep and brainstem cavernomas: a consecutive 8-year series. J Neurosurg. 2003;99(1):31-37.

16.McCormick WF: Pathology of vascular malformations of the brain, in Wilson CB, Steihn BM (eds): Intracranial Arteriovenous Malformations. Baltimore,Williams \& Wilkins, 1984, pp 44-63.

17.Mizoi K, Yoshimoto T, Suzuki J. Clinical analysis of ten cases with surgically treated brain stem cavernous angiomas. Tohoku J Exp Med. 1992;166(2): 259-267.

18. Nimjee SM, Powers CJ, Bulsara KR: Review of the literature on de novo formation of cavernous malformations of the central nervous system after radiation therapy. Neurosurg Focus 21:e4, 2006.
19.Pozzati E, Acciarri N, Tognetti F, Marliani F, Giangaspero F: Growth, subsequent bleeding, and de novo appearance of cerebral cavernous angiomas. Neurosurgery 38:662-670, 1996.

20.Quinones-Hinojosa A, Lyon R, Du R, Lawton MT. Intraoperative motor mapping of the cerebral peduncle during resection of a midbrain cavernous malformation: technical case report. Neurosurgery. 2005;56(2 Suppl):E439.

21.Rigamonti D, Hadley MN, Drayer BP, Johnson PC, Hoenig-Rigamonti K, Knight JT, Spetzler RF: Cerebral cavernous malformations. Incidence and familial occurrence. N Engl J Med 319:343-347, 1988.

22.Robinson JR, Awad IA, Little JR: Natural history of the cavernous angioma. J Neurosurg 75:709-714, 1991.

23.Samii M, Eghbal R, Carvalho GA, Matthies C. Surgical management of brainstem cavernomas. J Neurosurg. 2001;95(5):825-832.

24.Vinas FC, Gordon V, Guthikonda M, Diaz FG. Surgical management of cavernous malformations of the brainstem. Neurol Res. 2002;24(1): 61-72.

25.Zabramski JM, Wascher TM, Spetzler RF, et al. The natural history of familial cavernous malformations: results of an ongoing study. J Neurosurg. 1994;80(3):422432.

26.Wang CC, Liu A, Zhang JT, Sun B, Zhao YL. Surgical management of brainstem cavernous malformations: report of 137 cases. Surg Neurol.

2003;59(6):444-454.

27.Ziyal IM, Sekhar LN, Salas E, Sen C. Surgical management of cavernous malformations of the brain stem. Br J Neurosurg. 1999;13(4):366-375. 